\title{
Two-Stage Stochastic Unit Commitment Model Including Non-Generation Resources with Conditional Value-at-Risk Constraints
}

\author{
Yuping Huang a , Qipeng P. Zheng ${ }^{\mathrm{a}}$, Jianhui Wang ${ }^{\mathrm{b}}$ \\ ${ }^{a}$ Dept. of Industrial Engineering and Management Systems, University of Central Florida, Orlando, FL 32816, USA \\ ${ }^{b}$ Argonne National Laboratory, Argonne, IL 60439, USA
}

\begin{abstract}
This paper presents a two-stage stochastic unit commitment (UC) model, which integrates non-generation resources such as demand response (DR) and energy storage (ES) while including risk constraints to balance between cost and system reliability due to the fluctuation of variable generation such as wind and solar power. This paper uses Conditional Value-at-Risk (CVaR) measures to model risks associated with the decisions in a stochastic environment. In contrast to chance-constrained models requiring extra binary variables, risk constraints based on CVaR only involve linear constraints and continuous variables, making it more computationally attractive. The proposed models with risk constraints are able to avoid over-conservative solutions but still ensure system reliability represented by loss of loads. Then numerical experiments are conducted to study the effects of non-generation resources on generator schedules and the difference of total expected generation costs with risk consideration. Sensitivity analysis based on reliability parameters is also performed to test the decision preferences of confidence levels and load-shedding loss allowances on generation cost reduction.
\end{abstract}

Keywords: Stochastic Unit Commitment, Demand Response, Energy Storage, Conditional Value-at-Risk, Benders Decomposition, Sensitivity Analysis

\section{Introduction}

Stochastic unit commitment (SUC) is an effective modeling technique and it has been introduced as a promising tool to deal with power generation problems involving uncertainties $[1,2,3,4,5,6]$. SUC assumes scenario-based uncertainty in unit commitment problems, i.e. it captures the uncertainty and variability of the underlying factors by simulating a large number of scenarios. One of prominent factors is the high penetration of renewable energy to current power systems, which brings a lot of uncertainties on energy supply and transmission. Considering one of renewable energy resources like wind energy, the forecasting errors or intermittent energy supply in net load will cause conventional power plants to ramp up/down frequently to ensure their energy outputs satisfy real-time demand levels. Therefore, on one side, non-generation resources, e.g., demand response (DR) and energy storage (ES), have been well develope$\mathrm{d}$ and facilitate the expansion of renewable energy's usage. On the other side, management techniques for energy systems can be used effectively to ensure the smooth integration of existing power plants with renewable energy outputs [7] as well as power system reliability. This paper aims to investigate the unit commitment scheduling cooperated with non-generation resources and risk control so as to improve power system reliability and reduce cost. The main uncertainties in consideration of this paper include renewable energy output and demand response. This real-world problem is formulated through a two-stage stochastic mixed integer program. 
On one hand, energy storage is one of typical non-generation resources and a feasible solution to facilitate the integration of wind power generation. The main advantage is that it is able to provide electricity supply when the peak demands occur to be greater than generation capacities in a power system, or the generation costs are extremely high. Since the storage devices can store or release energy based on operations and demands, the incorporation of ES can increase the flexibility of power supply systems and decrease total costs at the same time. Some literature has discussed the economic value of ES investments, system-economic evaluations [8], optimal size and capacity for ES systems [9, 10], and stochastic operation management with ES on micro grid [11]. Recently, there are three main large-scale energy storage technologies, including pumped hydro accumulation storage (PAC), underground PAC and compressed air energy storage (CAES). Most studies of energy storage focus on CAES in the areas of economic value of investments, system-economic perspectives, technical challenges to the integration of wind power with power systems, and production planning $[12,13]$. In most of the optimization models, energy storage is introduced as time-dependent multi-period storage constraints. Senjyu et al. [14] discuss the thermal UC problem consisting of generalized energy storage systems (ESS) and solve the model by extended priority list. Daneshi and Srivastva [8] develop enhanced security-constrained UC with wind generation and CAES, and conduct the comprehensive analysis of CAES on economics, peak-load shaving and wind curtailment. Except to the function of peak shaving provided by ESS, the primary reserve requirements and their combined provision are investigated via economic assessment [15].

On the other hand, demand response mechanisms have been proposed and praticed for several years to encourage consumers to reduce power consumption during on-peak hours and increase uses at off-peak hours or the times of high production. Since there exist unavoidable forecast errors for day-ahead wind resource, this increases re-dispatch costs and loss of load events. Sioshansi [16] discusses the introduction of demand response by real-time pricing in order to mitigate these wind integration costs. Zhao and Zeng [17] also proposed a two-stage robust optimization model for UC with DR in the integration of wind energy and solved the problem by a novel cutting plane algorithm. On one hand, the effect of demand response in an isolated system with wind integration has been studied in [18]. DR-based reserve capacity has also been proved to be an effective mechanism to accommodate the uncertainty of wind generation, which has been studied by the extension of security-constrained unit commitment model with DR and performing simulation tests [19]. On the other hand, deterministic and stochastic security approaches were compared for energy and spinning reserve scheduling in presence of DR, where stochastic approach was shown to achieve a lower system cost and load shedding [20]. Later, Madaeni and Sioshansi [21] examined the effectiveness of stochastic programming and demand response on the reductions of wind uncertainty costs. Their empirical studies showed a stochastic program with DR brings more benefits significantly. Of the many modeling approaches of demand response, the method based on price elasticity matrix (PEM) will be utilized in our study. Although there are possibly some forecast errors existing in PEM, it is relatively easy to forecast loads which follow a specific end-user type. It is a good approximation for demand response and has been studied in [22]. The other benefit of this method offers easy incorporation with optimization models and produces sufficient results as well.

To limit the likelihood of load losses due to uncertainties, risk management has been merging to daily operations of power generation. Chance-constrained optimization models have been developed to deal with uncertain wind power output [23], uncertain load [24] and transmission network expansion planning [25]. Chance constraints are equivalent to constraints that bound the risk measure Value-at-risk (VaR). Another tighter risk measure defined upon $\mathrm{VaR}$ is conditional value-at-risk $(\mathrm{CVaR})$. As popular risk measures, VaR and CVaR have been widely used in financial risk management [26, 27, 28]. Compared to VaR based models, CVaR based models are less computationally demanding due to the fact that modeling CVaR only 
requires linear constraints and continuous variables. We thus introduce CVaR to our SUC model to maintain system reliability at various levels.

Compared to the recent works of stochastic programming approaches on unit commitment problems (e.g., [29, 30, 31, 21]), the main contributions of this study are summarized as follow:

1. A comprehensive two-stage stochastic mixed intger programming model for unit commitment with risk constraints based on CVaR is developed to control risk of loss of loads while including nongeneration resources. The proposed optimization model helps to satisfy real-time demands and minimize the total operation costs with the support of non-generation resources. The model can help balance between expected cost and risks of load losses.

2. A modified Benders' Decomposition algorithm is applied to solve for this CVaR-based model and reduce computation times.

3. Numerical experiments are conducted to find out optimal unit commitment solutions and compare the effects of the risk resilience of non-generation resources on power generation. Sensitivity analyses are also carried out to evaluate reliability parameters on reducing the generation costs.

The remainder of this paper is organized as follows. Section 2 discusses the mathematical formulations for risk-constrained unit commitment including demand response constraints, energy storage constraints, the integrated model with CVaR risk measures and solution approach. Section 3 provides numerical examples for the 7-bus system and the 118-bus system, respectively, and discusses their computational results and sensitivity analyses. Section 4 concludes the paper.

\section{Mathematical Formulation}

In this paper, we formulate a two-stage stochastic programming model for the unit commitment problem under uncertainties. Commitment decisions (here-and-now) are assumed to be made a day ahead, which is considered as the first stage. These decisions need to be able to accommodate the real-time situations with uncertain demands and renewable energy outputs. In order to model uncertainties, we use discrete scenarios within the set $\Xi$. Simulation techniques are used to generate different scenarios in real time. The second stage is addressing the real-time decisions (dispatch, transmission, etc.) under all scenarios, which are captured by $|\Xi|$ sets of constraints and variables. In addition, the second stage is linked with day-ahead unit commitment decisions through dispatch constraints. The unit commitment decisions on the first stage only contain binary decision variables and are determined before the uncertain demands and renewable generation outputs realized. The second stage handles the issues regarding economic dispatch, power transmission, demand response and energy storage after the uncertainties unfold. To incorporate reliability explicitly using scenario information, we also introduce the risk constraints in the second stage. A comprehensive nomenclature of sets, parameters, and variables in this paper is attached after the last section.

The objective function (1) is composed of two parts. The first part is the total start-up and shutdown cost for day-ahead unit commitment decisions (in first stage) since we assume no reschedules of units in real time. The second part is the total cost associated with the second stage, which is the expected fuel cost. Because we are using discrete scenarios, it is a weighted average of the fuel costs of all scenarios. The objective function of this two-stage stochastic model is presented as follows,

$$
\begin{gathered}
\sum_{g \in G} \sum_{t \in T}\left(S U_{g t} v_{g t}+S D_{g t} w_{g t}\right) \\
+\sum_{\xi \in \Xi} \operatorname{Prob}^{\xi} \sum_{g \in G} \sum_{t \in T} F_{g}\left(p_{g t}^{\xi}\right) \\
3
\end{gathered}
$$


Note the first part of the objective function is deterministic (determined by here-and-now decisions) while the second term is an expected cost for electricity dispatch. The fuel cost is actually a quadratic function of the dispatch/production level, $p$, i.e., for generator $g, F_{g}(p)=a+b p+c p^{2}$, where $a, b$ and $c$ are usually positive coefficients. An example of these parameters can be found in Table 3. Because the fuel cost function is nonlinear (which can complicate the computation with the presence of binary decisions), a piecewise linear approximation is used to yield very close solutions instead of directly solving the mixed integer quadratic problem. In order to obtain the piecewise linear approximation of the fuel cost function, SOS techniques are used to replace the original function $F_{g}(p)$ by $\sum_{k=1}^{K} C_{k} \lambda_{k}$ with additional constraints, $\left\{p=\sum_{k=1}^{K} \Delta_{k} \lambda_{k}, \sum_{k=1}^{K} \lambda_{k}=u, \lambda_{k} \geq 0, k=1, \ldots, K\right\}$, where $u$ is the commitment status of generator $g$, and $\Delta_{k}$ and $C_{k}$ are parameters used to approximate the quadratic curve. For more details please refer to [32]. Hence, with the piecewise linear approximation, we have a purely mixed integer program. In the following subsections, we will explain the different sets of constraints and variables for the two-stage stochastic mixed integer linear programming model.

\subsection{Unit Commitment and Dispatch Formulation}

As the here-and-now decision making part, unit commitment is to determine the operating status of generation units in a power system to meet the next day's demands. But the first stage mainly involves the constraints on the commitment status of generators at different times. The following constraints (2)-(5) represent the requirements for minimum up time, minimum down time, startup action, and shutdown action of each unit at each time period, respectively.

$$
\begin{array}{ll}
u_{g t}-u_{g(t-1)} \leq u_{g \tau} & \forall g \in G, t \in T, \\
u_{g(t-1)}-u_{g t} \leq 1-u_{g \tau} & \tau=t, \ldots, \min \left\{t+L_{g}-1,|T|\right\} \\
v_{g t} \geq u_{g t}-u_{g(t-1)} & \forall g \in G, t \in T, \\
w_{g t} \geq-u_{g t}+u_{g(t-1)} & \tau=t, \ldots, \min \left\{t+l_{g}-1,|T|\right\} \\
u_{g t}, v_{g t}, w_{g t} \in\{0,1\} & \forall g \in G, t \in T \\
& \forall g \in G, t \in T
\end{array}
$$

where three binary variables, $u_{g t}, v_{g t}, w_{g t}$, are defined as commitment decision, startup action and shutdown action of unit $g$ at period $t$ respectively. $L_{g}$ and $l_{g}$ are minimum-on time and minimum-down time, respectively.

As the wait-and-see decision making part, economic dispatch is to fulfill system operations subject to available resources and then to achieve the optimal output for demand satisfactions. The dispatch or generation levels are treated as the wait-and-see decisions given the day-ahead unit commitment status. Their function is mainly reflected in the generation lower limit (7) and the upper limit constraint (8), and the ramping up and down limit constraint (9). In addition, there exist some possibilities at specific generators to increase power output by spinning reserve, which are shown in the constraints of satisfying system spinning reserve (10) and spinning reserve limit (11). The nonnegative restriction of generator dispatch is ensured by constraint (12). Note that there are $|\Xi|$ sets of these decision variables and constraints, with each set 
representing a scenario indexed by $\xi$. The constraints are shown as follows,

$$
\begin{aligned}
& P_{g}^{\min } u_{g t} \leq p_{g t}^{\xi} \\
& p_{g t}^{\xi}+s_{g t}^{\xi} \leq P_{g}^{\max } u_{g t} \\
& -R D_{g} \leq p_{g t}^{\xi}-p_{g t-1}^{\xi} \leq R U_{g} \\
& \sum_{g \in G_{i}} s_{g t}^{\xi} \geq R S_{i t} \\
& 0 \leq s_{g t}^{\xi} \leq S_{g}^{\max } \\
& p_{g t}^{\xi} \geq 0
\end{aligned}
$$

$$
\begin{aligned}
& \forall g \in G, t \in T, \xi \in \Xi \\
& \forall g \in G, t \in T, \xi \in \Xi \\
& \forall g \in G, t \in T, \xi \in \Xi \\
& \forall i \in N, t \in T, \xi \in \Xi \\
& \forall g \in G, t \in T, \xi \in \Xi \\
& \forall g \in G, t \in T, \xi \in \Xi
\end{aligned}
$$

\subsection{Demand Response Formulation}

Demand side management or demand response (DR) can be an effective tool to mitigate the peak load or peak-to-average ratio. To avoid the extensive use of the expensive peak load plants, system operators take into account responsive demands to price signals. This fact can be approximated by a set of linear constraints using a price elasticity matrix when price variation is small as in [22]. In these linear constraints, the shifted demand at time $t$ is an affine function of the price variations in all other time periods, where the constant term is the reference demand at time $t$. Actually, the major uncertainty on the demand sides is the responding behaviors of end consumers (modeled by the price elasticity matrix $\mathbf{E}_{i}^{\xi}$ ) on varying electricity prices. The real-time demand comprises the forecasted demand and the demand adjustment caused by changes of electricity prices from the benchmark price $\mathbf{Q}_{i}^{\xi}$ (by multiplying the price elasticity matrix $\mathbf{E}_{i}^{\xi}$ with the price variation vector $\mathbf{q}_{i}^{\xi}-\mathbf{Q}_{i}^{\xi}$ ). Although renewable energy is considered as another uncertainty source in our computational model, we assume that renewable energy output is independent of DR and electricity price over the planning horizon. Thus, the demand adjustment is only affected by the uncertain price elasticity matrix and varying electricity prices. Within a scenario, we also assume the total sum of demands at all time period at any location/bus is a constant. This is guaranteed by the loss price elasticity matrix $\mathbf{E}_{i}^{\xi}$ for each bus, in which the summation of each column equals to zero [33, 34].

Constraint (13) demonstrates that the real-time demand is equal to the summation of forecasted benchmark demand and elastic demand based on the price elasticity matrix. Besides, the electricity price constraint (14) controls the real-time price fluctuation in a reasonable range. These two constraints are shown as follows,

$$
\begin{aligned}
\mathbf{y}_{i}^{\xi}=\mathbf{D}_{i}^{0}+\mathbf{E}_{i}^{\xi}\left(\mathbf{q}_{i}^{\xi}-\mathbf{Q}_{i}^{\xi}\right) & \forall i \in N, \xi \in \Xi \\
\alpha \mathbf{Q}_{i}^{\xi} \leq \mathbf{q}_{i}^{\xi} \leq \gamma \mathbf{Q}_{i}^{\xi} & \forall i \in N, \xi \in \Xi
\end{aligned}
$$

where $\mathbf{y}_{i}^{\xi}$ is the real-time demand vector at node $i$ under scenario $\xi ; \mathbf{D}_{i}^{0}$ is the hourly benchmark/reference demand vector forecasted in day ahead; $\mathbf{E}_{i}^{\xi}$ is the uncertain price elasticity matrix reflecting demand change rates due to varying electricity prices; $\mathbf{q}_{i}^{\xi}$ is the real-time electricity price vector; $\mathbf{Q}_{i}^{\xi}$ is the benchmark/reference electricity price vector at node $i$ in scenario $\xi$. In the above linear constraints, $\mathbf{y}_{i}^{\xi}$ and $\mathbf{q}_{i}^{\xi}$ are the decision variables, and the others are given parameters. Each of the vectors are composed of the elements of different time periods, for example, $\mathbf{y}_{i}^{\xi}=\left[y_{i t}^{\xi}, \forall t \in T\right]^{T} . \alpha$ and $\gamma$ are coefficients used to bound the possible electricity prices, which is necessary to maintain the validity of the linear approximation of demand response [22]. 


\subsection{Energy Storage Formulation}

In the current electrical power systems, electricity has to be used immediately according to the physical law on power circuits. This fact leads to many issues concerning the power systems, e.g., high redundancy, supply and demand imbalance, etc. Meanwhile, the renewable energy penetration continues growing and greatly increases the difficulty of power system operations. With the advancement of energy storage devices, these issues can be mitigated using these devices in the power systems. We formulate a set of energy storage (ES) constraints to address the accumulators status, power saving and dispatch at each period of a scenario. Constraint (15) indicates energy balance for each accumulator; the other constraints (16) and (17) indicate the available dispatch level and power storage capacity, respectively.

$$
\begin{array}{ll}
r_{i t}^{\xi}=r_{i t-1}^{\xi}+v_{i t-1}^{\xi}-x_{i t-1}^{\xi} & \forall i \in N, t \in T, \xi \in \Xi \\
0 \leq x_{i t}^{\xi} \leq r_{i t}^{\xi} & \forall i \in N, t \in T, \xi \in \Xi \\
0 \leq r_{i t}^{\xi} \leq \kappa_{i} & \forall i \in N, t \in T, \xi \in \Xi \\
v_{i t}^{\xi} \geq 0 & \forall i \in N, t \in T, \xi \in \Xi
\end{array}
$$

where $r_{i t}^{\xi}$ is the total remaining power in storage facilities of unit $i$ at time $t, v_{i t-1}^{\xi}$ is the power storage at node $i$ in period $t$ of scenario $\xi, x_{i t-1}^{\xi}$ is the renewable energy dispatch amount at node $i$ in period $t$ of scenario $\xi$ and $\kappa_{i}$ is the maximum storage capacity at node $i$ under scenario $\xi$. Note that $N$ can be replaced by a subset $N^{\prime} \subset N$, because ES devices are not necessarily at every bus.

\subsection{Transmission Formulation}

In our application, we formulate the power transmission using an approximation of power flows. Generally, Kirchhoff's current and voltage laws apply to interconnected electrical network (e.g., a electrical power grid), and are used to find out electricity characteristics of transmission and distribution systems. To consider possible loss from load-shedding, the traditional DC approximation of Kirchhoff's current law (KCL) constraints are modified to involve the loss that occurs at location $i$ at time $t$ under scenario $\xi, l_{i t}^{\xi}$, shown in constraint (19). In many cases, we can restrict $l_{i t}^{\xi}$ to zero. And a DC approximation of Kirchhoff's voltage law is expressed in constraint (20).

$$
\begin{array}{ll}
\sum_{(i, j) \in A_{i}^{+}} f_{i j t}^{\xi}-\sum_{(j, i) \in A_{i}^{-}} f_{j i t}^{\xi}=\sum_{g \in G_{i}} p_{g t}^{\xi}+R_{i t}^{\xi}-D_{i t}^{0}+l_{i t}^{\xi}, & \forall i \in N, t \in T, \xi \in \Xi \\
\left(f_{i j t}^{\xi}-f_{j i t}^{\xi}\right)-B_{i j t}^{\xi}\left(\beta_{i t}^{\xi}-\beta_{j t}^{\xi}\right)=0, & \forall(i, j) \in A, t \in T, \xi \in \Xi \\
f_{i j t}^{\xi}, l_{i t}^{\xi} \geq 0, & \forall(i, j) \in A, i \in N, t \in T, \xi \in \Xi
\end{array}
$$

where $f_{i j t}^{\xi}$ is an unrestricted variable representing a bi-direction flow between bus $i$ and bus $j ; A_{i}^{+}$and $A_{i}^{-}$ denote the set of flow starting at bus $i$ and the set of flow ending at bus $i$, respectively. As the absence of demand response program, the real-time demand is equivalent to the benchmark demand $D_{i t}^{\xi 0}$.

When ES devices are connected to the grid at some nodes and DR programs are implemented, the transmission constraints are also revised to adopt the process of energy storage and dispatch. In fact, the operation of energy storage can be considered as power consumption from the bus and the operation of dispatch can be considered as power supply to the electric grid, the amounts of which are represented by $v$ and $x$, respectively. The implementation strategy of UC combined with DR and ES is able to affect the total 
expected generation cost under their joint actions. Therefore, the KCL constraint is modified as follows,

$$
\begin{gathered}
\sum_{(i, j) \in A_{i}^{+}} f_{i j t}^{\xi}-\sum_{(j, i) \in A_{i}^{-}} f_{j i t}^{\xi}=\sum_{g \in G_{i}} p_{g t}^{\xi}+\rho_{i} x_{i t}^{\xi}+R_{i t}^{\xi}+l_{i t}^{\xi}-v_{i t}^{\xi}-y_{i t}^{\xi}, \\
\forall i \in N, t \in T, \xi \in \Xi
\end{gathered}
$$

where $\rho_{i}$ addresses the ES efficiency which is determined by device properties and $y_{i t}^{\xi}$ is the real-time demand influenced by DR.

\subsection{Risk Constraints}

In scenario-based two-stage stochastic programming models, usually a large number of simulated scenarios (e.g., wind outputs, nodal demands) are used. Since the stochastic programming formulation includes all scenarios, the optimal solutions might be very overconservative with high total cost because feasible solutions need to compensate much for the extreme scenarios. On the other hand, we also need to maintain a certain level of system reliability. Hence we need to balance between the total cost and system reliability. To this end, chance or risk constraints are usually introduced in the stochastic programming models for this tradeoff.

Risks in stochastic unit commitment usually are linked with loss of load since a reliable system should be able to meet as much demand as it can. Hence loss of load probability (LOLP) is usually required to stay below an allowed level in many previous approaches [24, 35]. LOLP can be directly modeled by chance constraints, which is equivalent to bound a $\theta$-level Value at Risk (VaR) of the loss of load, where $\theta$ is usually a value close to 1 . Different policies regarding how to aggregate loss of load (e.g., total loss over all time periods v.s. loss of each time period) can be used to define the LOLP constraints [23]. In this paper, we are trying to bound the risks associated with each individual time period. Let $L(x, Y)$, a random variable, be the loss function (e.g., total loss of all buses at a time period), where $x$ are the aggregated decision vector and $Y$ is the random vector (e.g., wind outputs). $\operatorname{VaR}_{\theta}[L(x, Y)]$ is the $\theta$-level Value-at-Risk (VaR) of the loss of load function $L(x, Y)$. It is also the $\theta$-level quantile of the random variable $L(x, Y)$, which can be defined as follows,

$$
\operatorname{VaR}_{\theta}[L(x, Y)]=\min _{l}\{l \mid \operatorname{Prob}(L(x, Y) \leq l) \geq \theta\} .
$$

Chance constraints are equivalent to bound $\operatorname{VaR}_{\theta}[L(x, Y)]$ above by $\bar{l}$, which is the maximum tolerable loss of load, usually set as 0 . Since VaR constraints involve binary variables and big M to select good/bad scenarios, it will cause many computational difficulties when solving the chance-constrained programs especially with large number of scenarios. Approximation algorithms such as Sample Average Approximation are used to solve chance-constrained stochastic unit commitment problems [23, 35].

In this paper, we choose to bound another risk of load loss, Conditional Value at Risk (CVaR), also named as Average Value at Risk (AVaR) or Expected Tail Loss (ETL). It is a coherent risk measure widely used in many areas, e.g., financial and risk management [36], natural gas system expansion planning [37], stochastic network optimization [38]. CVaR constraints only involve continuous variables and linear constraints, and then are computationally friendly even with a large number of scenarios. In addition, the optimal solution of CVaR-based models also provide information of corresponding VaR measure because $\mathrm{CVaR}$ is the conditional expectation of the loss function given that the loss is beyond $\operatorname{VaR}_{\theta}[L(x, Y)]$. Hence 
the CVaR constraints also include VaR definition shown as follows,

$$
\begin{aligned}
\min _{l}\{l \mid \operatorname{Prob}(L(x, Y) \leq l) \geq \theta\} & =\eta \\
\mathbb{E}\{L(x, Y) \mid L(x, Y) \geq \eta\} & \leq \bar{\phi}
\end{aligned}
$$

where $\mathbb{E}$ refers to the expectation, and $\eta$ is $\operatorname{VaR}_{\theta}[L(x, Y)]$, and $\bar{\phi}$ is the maximum tolerable loss for CVaR. Note that this does not mean maximum tolerable loss for $\eta$ is $\bar{\phi}$. In fact $\operatorname{VaR}_{\theta}[L(x, Y)]$ is bounded by a loss smaller than $\bar{\phi}$. We refer the readers to $[26,28]$ for further details including the discussion between VaR and CVaR, and the constraints to represent them.

For the stochastic unit commitment problem, we choose to bound the CVaR linked to the total load loss of all bus at each time period. Because we have to model the expectation beyond $\operatorname{VaR}_{\theta}[L(x, Y)]$, we need to split the loss of time period $t$ into two parts $\eta_{t}$ and $\zeta_{t}^{\xi}$ as shown in (24). $\eta_{t}$ represents the actual $\operatorname{VaR}_{\theta}[L(x, Y)]$ at time $t$, and $\zeta_{t}^{\xi}$ represent the loss beyond the value at risk in scenario $\xi$ because both of them only take nonnegative values. On the left hand side of constraint (25), these two are combined again to calculate the conditional expectation, which is $\mathrm{CVaR}_{\theta}[L(x, Y)]$. Then it is bounded above by $\bar{\phi}$, a loss allowance parameter. The risk constraints based on CVaR are shown as follows,

$$
\begin{array}{ll}
\sum_{i \in I} l_{i t}^{\xi} \leq \eta_{t}+\zeta_{t}^{\xi}, & \forall t \in T, \xi \in \Xi \\
\eta_{t}+(1-\theta)^{-1} \sum_{\xi \in \Xi} \operatorname{Prob}^{\xi} \zeta_{t}^{\xi} \leq \bar{\phi}, & \forall t \in T \\
\eta_{t} \geq 0, \zeta_{t}^{\xi} \geq 0, & \forall t \in T, \xi \in \Xi
\end{array}
$$

\subsection{SUCR-DR-ES Model}

We then propose the following integrated model for the security-constrained unit commitment with risk control including DR and ES constraints at the same time, called SUCR-DR-ES. The integrated model includes UC constraints (2)-(12), DR constraints (13)-(14), ES constraints (15)-(18), transmission constraints (20)-(22), and risk constraints (24)-(26).

$$
\begin{aligned}
\text { [SUCR-DR-ES]: } \min & \sum_{g \in G} \sum_{t \in T}\left(S U_{g t} v_{g t}+S D_{g t} w_{g t}\right) \\
& +\sum_{\xi \in \Xi} \operatorname{Prob}^{\xi} \sum_{g \in G} \sum_{t \in T} F_{g}\left(p_{g t}^{\xi}\right) \\
\text { s.t. } & (2)-(12),(13)-(14),(15)-(18) \\
& (20)-(22),(24)-(26)
\end{aligned}
$$

\subsection{Solution approach}

When a large number of scenarios are included in the stochastic models, Benders decomposition can be utilized to address the computational issues, especially for the special structures of mixed integer linear programs [39]. While applying Benders decomposition, the original SUCR-DR-ES problem is decomposed into a relaxed master problem and multiple subproblems based on each scenario. In the classical Benders decomposition algorithm, Benders' cuts are constructed using the optimal dual solutions of subproblem in each iteration. Then they are added to the relaxed master problem (RMP) for the next iteration, so as to improve the lower bound on the original problem. 
In general cases, an original model is decomposed to an integer program of RMP and a linear program of subproblem. According to this decomposition strategy, SUCR-DR-ES is naturally decomposed into the RMP only with unit commitment constraints and the subproblem with the resting constraints. However, this decomposition can produce low-density cuts that only involve single decision variable $\mathbf{u}$ and practically cause a slow convergence. In addition, in the subproblem, all scenarios are coupled together by the CVaR constraints, which could potentially restrict the use of parallel computing resources. We then choose an alternative decomposition strategy to handle this issue, in the way of increasing the density of Benders cuts. In the fact that a coupling constraint (25) appears in the CVaR constraints, this coupling structure is not easy to handle on decomposition algorithms. Thus all CVaR constraints are placed on RMP, and only the incumbent solutions $(\mathbf{u}, \mathbf{I})$ will be passed to $\mathbf{S P}^{\xi}$. In doing so, multiple Benders cuts are generated including loss variable $\mathbf{I}$ and able to restrict equivalent or more solution space of RMP in one iteration. In addition, we have multiple uncoupled subproblems, which can take advantage of parallel computing resources to reduce computing times. Let $\pi^{\xi}$ be an unrestricted variable to represent the minimum total fuel cost in a scenario. The relaxed master problem includes unit commitment and CVaR constraints, shown as,

$$
\begin{aligned}
{[\mathbf{R M P}]: \min } & \sum_{g \in G} \sum_{t \in T}\left(S U_{g t} v_{g t}+S D_{g t} w_{g t}\right)+\sum_{\xi \in \Xi} \operatorname{Prob}^{\xi} \pi^{\xi} \\
\text { s.t. } \quad & (2)-(6),(24)-(26), \\
& \mathbb{F}\left(u_{g t}, l_{i t}^{\xi}, \pi^{\xi}\right) \geq 0, \forall \xi \in \Xi
\end{aligned}
$$

where constraint $\mathbb{F}\left(u_{g t}, l_{i t}^{\xi}, \pi^{\xi}\right) \geq 0$ stands for Benders' cuts associated with the commitment variable $u_{g t}$ and loss variable $l_{i t}^{\xi}$. These cuts are generated based on solutions from the subproblem based on one scenario.

To avoid the case of infeasible subproblems, the subproblem formulation of SUCR-DR-ES adopts the Big-M method, in which nonnegative artificial variables are introduced (with a big penalty in the objective function) to insure $\mathbf{S P}^{\xi}$ maintain the feasibility given any first-stage decision. The artificial variable $\omega_{i t}$ is introduced to the system spinning reserve constraint (10); the artificial variables $o_{i t}^{+}$, and $o_{i t}^{-}$are introduced to the KCL transmission constraint (22). If any of the artificial variables is not equal to zero, the objective function of $\mathbf{S P}^{\xi}$ then will be penalized with a large number $M$ associated with artificial variables. Given the incumbent solutions $(\mathbf{u}, \mathbf{I})$, the subproblem (27) is to optimize the generation dispatch, subject to scenarioindependent inequalities. The subproblems with Big-M method is shown as follows,

$$
\begin{aligned}
{\left[\mathbf{S P}^{\xi}\right]: \min } & \sum_{g \in G} \sum_{t \in T} F_{g}\left(p_{g t}^{\xi}\right)+M \sum_{t \in T} \sum_{i \in N}\left(\omega_{i t}+o_{i t}^{+}+o_{i t}^{-}\right) \\
\text {s.t. } \quad & (9),(11)-(18),(20)-(21) \\
& p_{g t}^{\xi} \geq P_{g}^{\min } \hat{u}_{g t}, \forall g \in G, t \in T \\
& p_{g t}^{\xi}+s_{g t}^{\xi} \leq P_{g}^{\max } \hat{u}_{g t}, \forall g \in G, t \in T \\
& \sum_{g \in G_{i}} s_{g t}^{\xi}+\omega_{i t} \geq R S_{i t}, \forall i \in N, t \in T \\
& \sum_{(i, j) \in A_{i}^{+}} f_{i j t}^{\xi}-\sum_{(j, i) \in A_{i}^{-}} f_{j i t}^{\xi}+o_{i t}^{+}-o_{i t}^{-} \\
& =\sum_{g \in G_{i}} p_{g t}^{\xi}+\rho_{i} x_{i t}^{\xi}+R_{i t}^{\xi}-v_{i t}^{\xi}-y_{i t}^{\xi}+\hat{l}_{i t}^{\xi}, \forall i \in N, t \in T
\end{aligned}
$$

where only the second-stage constraints are included such as economic dispatch, non-generation resources 
and power transmissions.

We define a series of dual variables, i.e. $\varepsilon_{g t}^{\xi}, \rho_{g t}^{\xi}, \chi_{g t}^{\xi}, \sigma_{g t}^{\xi}, \tau_{i t}^{\xi}, v_{g t}^{\xi}, \lambda_{i t}^{\xi}, \mu_{i t}^{\xi}, v_{i t}^{\xi}, \vartheta_{i t}^{\xi}, \varphi_{i t}^{\xi}$, corresponding to the constraints (27b), (27c), (9), (9), (27d), (11), (13), (14), (14), (17), (27e). For example, dual variables $\chi_{g t}^{\xi}$ and $\sigma_{g t}^{\xi}$ correspond to the ramping up and ramping down in (9), respectively. After solving the $\mathbf{S P}^{\xi}$, one can obtain the optimal dual values corresponding to the above constraints. These dual values for one scenario are then used to construct an optimality cut $\mathbb{F}\left(u_{g t}, l_{i t}^{\xi}, \pi^{\xi}\right)$, which is presented in (28).

$$
\begin{aligned}
\pi^{\xi} \geq & \sum_{g \in G} \sum_{t \in T} \hat{\varepsilon}_{g t}^{\xi} P_{g}^{\min } u_{g t}+\sum_{g \in G} \sum_{t \in T} \hat{\rho}_{g t}^{\xi} P_{g}^{\max } u_{g t}+\sum_{g \in G} \sum_{t \in T} \hat{\chi}_{g t}^{\xi} R U_{g} \\
& +\sum_{g \in G} \sum_{t \in T} \hat{\sigma}_{g t}^{\xi} R D_{g}+\sum_{i \in N} \sum_{t \in T} \hat{\tau}_{i t}^{\xi} R S_{i t}+\sum_{g \in G} \sum_{t \in T} \hat{v}_{g t}^{\xi} S_{g}^{\max } \\
& +\sum_{i \in N} \sum_{t \in T} \hat{\lambda}_{i t}^{\xi}\left(D_{i t}^{0}-E_{i t}^{\xi} Q_{i t}^{\xi}\right)+\sum_{i \in N} \sum_{t \in T} \hat{\mu}_{i t}^{\xi} \alpha Q_{i t}^{\xi}+\sum_{i \in N} \sum_{t \in T} \hat{v}_{i t}^{\xi} \gamma Q_{i t}^{\xi} \\
& +\sum_{i \in N} \sum_{t \in T} \hat{\vartheta}_{i t}^{\xi} \kappa_{i}+\sum_{i \in N} \sum_{t \in T} \hat{\varphi}_{i t}^{\xi}\left(R_{i t}^{\xi}+l_{i t}^{\xi}\right)
\end{aligned}
$$

In the classic Benders' decomposition method, all Benders cuts generated from each iteration are appended to RMP directly and then RMP is solved for optimality again. This way of adding the cuts to RMP iteratively keeps increasing the size of active constraint set, but do not guarantee to provide stronger restriction of the solution space. It probably yields the considerable rework and leads to the slow convergence of the algorithm. In this paper, we implement Benders' Decomposition using CALLBACK function in CPLEX. The solution flowchart (Figure 1) explicitly addresses Benders' decomposition used to solve SUCR-DR-ES model by implementing CALLBACK function. The vector $\varsigma$ represents all continuous variables involved in the subproblems. Compared to the classical method, the Benders' decomposition with CALLBACK function has one of prominent advantages, where only violated cuts are chosen and added to RMP and other cuts are carried in a pool. In other words, this means is capable of maintaining the small size of RMP and applying a limited number of stronger or equivalent Benders's cuts.

The other advantage of implementing CALLBACK function is that RMP is solved only once. The whole process of solving RMP utilizes Branch-and-Bound-and-Cut algorithm. Meanwhile, Benders' cuts are generated at the branching nodes and added within the Branch-Bound-and-Cut algorithm. Particularly, only the most violated Benders' cuts are involved. The lower bound is being updated along with the RMP solving procedure (the branch-and-bound tree) where the upper bound also is created at a branching node after solving the $\mathbf{S P}^{\xi}$ for all scenarios. Until the RMP solving procedure is finished, the lower bound and upper bound are obtained and converged. Since the lower bound can be effectively improved with the help of Benders' cuts during a RMP solving procedure, it can avoid that the RMP is solved iteratively in the classical method, and thus the overall computation time is reduced.

\section{Computational Results}

To test the effects of reliability parameter variations, we perform the computational experiments to test the SUCR-DR-ES model described in section 2. In addition, we study the effects on risk resilience of using different non-generation resources. To this end, we also test another three models, namely, the SUCR model, SUCR-DR model and SUCR-ES model. They are all simplified versions of the SUCR-DRES model. For example, SUCR model does not include any DR and ES resources; SUCR-DR model only 
includes DR resources; SUCR-ES model only incorporates ES resources. Then their results are compared with the SUCR-DR-ES model based on the case studies with same inputs.

In the 7-bus system, four models are tested to compare the effects of their optimal schedules on the total thermal generation costs, based on a day ahead 100-scenario case. Additionally, we perform sensitivity analysis on reliability parameters, shadow price analysis and reliability parameter analysis to identify most affected range of cost increment as well as the relationship between objective value and the percentage change rate of cost increment. In the enhanced 118-bus system, we run each model at 10 different loss allowance cases with 7 different confidence levels to compare the strategies for using non-generation resources. It aims to verify the effectiveness of modified Bender's decomposition approach on solving our proposed models and find out risk management settings to reduce total costs. Normal distribution is assumed to generate renewable energy and demand change scenarios. All models are coded in C++ while solved by CPLEX 12.5. All experiments are implemented on a PC Dell OPTIPLEX 980 with Intel Core i7 vPro at $2.80 \mathrm{GHz}$ and $8 \mathrm{~GB}$ memory in a Windows 7 operating system.

\subsection{Seven-Bus System}

The 7-bus system includes one wind farm, four generators, five loads and ten transmission lines. The characteristics of buses, the wind farm, thermal units and transmission lines are shown in Table $1-4$, respectively. The renewable energy resource is located at Bus 1 with a generating capacity of $100 \mathrm{MW}$. In many of the existing research efforts on stochastic UC with renewable energy resources, wind power output is assumed to be normally distributed (e.g., [40, 5, 23]). Following this stream, we also use normal distribution to generate the wind power output scenarios, although our models and algorithms can easily take on data generated from other distributions. The hourly renewable energy output falls in the range of $[5,100]$ MW and is produced by adding the random number from normal distribution generators in C++ to the hourly base load. The piecewise linear fuel cost function is used in the objective function. The estimated benchmark electricity prices are generated based on the pattern of hourly real-time locational marginal prices (LMP). The demand elasticity matrix includes the random load increase during 1 a.m. to 5 a.m. and the load reduction between 12 p.m. and 7 p.m., within the range of variation ratio, $[-1,1]$. The storage facilities are located at Bus 1, 2, 4 and 5 with corresponding storage capacities as shown in Table 1.

Firstly, we run all four models with $85 \%$ confidence level to show the effects of using different nongeneration resources or their combination on unit commitment scheduling and its total cost. The loss allowance from load-shedding, $\bar{\phi}$, is fixed to $5 \%$ of maximum hourly demand, whereas price velocity indicators $(\alpha, \gamma)$ are set to $(0.95,1.05)$. From the unit commitment results shown in Table 5 , it can be observed that all four models have G1 and G2 operate for the whole day and G3 off because it has very high fuel cost and startup/shutdown cost. The difference among power generation schedules occurs on G4. The SUCR model always requires G4 online to satisfy the demand and accommodate the volatility of renewable energy inputs. However, it is not necessary to keep G4 online at any period in a day when the DR program is implemented. In SUCR-DR results, G4 is off at $2 \mathrm{a} . \mathrm{m}$. and $3 \mathrm{a} . \mathrm{m}$. according to the known daily load shifting. The SUCR-ES model is more flexible as compared to SUCR-DR model, because it further reduces the G4's generation time, only from 8 a.m. to 9 p.m. As in the case of SUCR-DR-ES, the optimal schedule only requires $\mathrm{G} 4$ online for 12 hours between 8 a.m. to 8 p.m., and has the lowest total cost $\$ 50480.5$. It is clear that the combined effects of DR and ES improve the generation schedule most significantly in terms of the total cost. The system benefits not only from load-shedding and load-shifting to lower the usage of generating equipments and fuel consumption, but also the increased flexibility and reliability of power supply.

Secondly, we conduct another set of numerical tests regarding risk management settings in the riskconstrained stochastic unit commitment. Sensitivity analyses are performed with respect to the confidence 
level $\theta$ and the load-shedding loss allowance $\bar{\phi}$. The optimal cost variations are presented when we increase the confidence level $\theta$ from $60 \%$ to $99 \%$ and the percentage of load-shedding loss allowance $\bar{\phi}$ from $1 \%$ to $20 \%$ (defined as $[$ Loss Limit $/($ Max Total Demand $)] \times 100 \%)$. The optimal objective values for all four models with different confidence levels and loss allowances are shown in the three-dimensional diagram (Figure 2). One of horizontal axes represents the percentage of loss allowance, the other horizontal axis represents the confidence level, and the vertical axis represents the cost reduction percentage. In comparisons of the heights of each plane, the SUCR-DR-ES model can yield the smallest objective costs since it takes advantage of combined actions of DR and ES. When the reliability parameters $(\theta$ and $\bar{\phi})$ are altered, the SUCR-DR-ES model still yields the lowest expected generation costs compared to the other three models given the same risk/reliability parameters.

Thirdly, we discuss the shadow price analysis on the reliability parameters, i.e., confidence level and loss allowance. Because all models are mixed integer linear programs, the dual information (denoting the shadow prices) are not readily available. Instead, we use the approximated shadow price for loss allowance, defined as $\partial z^{*} / \partial \bar{\phi} \approx\left(z_{N}^{*}-z_{N-1}^{*}\right) /\left(\bar{\phi}_{N}-\bar{\phi}_{N-1}\right)$, where $z^{*}$ denotes the optimal total cost and $N$ is the index. It means the unit total cost reduction per increment of loss allowance. In addition, holding loss allowance fixed, the approximated shadow price of confidence level is defined as $\partial z^{*} / \partial \theta \approx\left(z_{N}^{*}-z_{N-1}^{*}\right) /\left(\theta_{N}-\theta_{N-1}\right)$. In this way, we can find out the change rate of optimal objective value with respect to the increment of decision parameter. It helps the decision makers (e.g., ISOs) clearly locate the levels of reliability parameters that can impact the optimal cost significantly, and therefore make the right choice of parameters.

Figure 3 shows the percentage change rates comparisons for four models at different confidence levels and loss allowances, respectively. In Figure 3a, the percentage change rate of total cost increment with respect to confidence level is calculated by the formula $\left[\left(z_{\theta}^{*}-z_{\theta-5 \%}^{*}\right) /\left(z_{\theta=99 \%}^{*} \times 5 \%\right)\right] \times 100 \%$. The percentage change rates of total cost increment for SUCR-DR form a sharp peak between $60 \%$ and $80 \%$ and flatten out from $85 \%$ confidence level. However, the SUCR-ES has an opposite trend where the percentage change rates hold steady until a big jump occurs after $85 \%$ confidence level. This observation demonstrates that the effective range of confidence levels works differently on different models. Generally, a higher confidence level used in models means that the generation system has higher reliability. Thus all models except SUCR-ES show an applicable advantage at the high confidence level $(\geq 85 \%)$ because they increase the optimal cost more slowly.

In the Figure 3b, the comparisons of the percentage change rates of total cost reduction aim to identify the intensity response for each model while increasing the loss allowance. The percentage change rate of total cost reduction is defined as $\left[\left(z_{\bar{\phi}-1 \%}^{*}-z_{\bar{\phi}}^{*}\right) /\left(z_{\bar{\phi}=0}^{*} \times 1 \%\right)\right] \times 100 \%$, which shows the relationships between the percentage change rate of total cost reduction and loss allowance by percentage. Since the total cost reduction percentage change rates are negative in our case studies, they are converted to positive values so that it's convenient for comparison and analysis. Both SUCR and SUCR-DR have significantly higher percentage change rates than the other two models in the instance of $1 \%$ loss allowance. This indicates that the SUCR and SUCR-DR are affected by the change of loss allowance more significantly, especially on the range of low loss allowance. When the loss allowance is greater than the regular loss cap (3\%), the models using DR, ES or both have similar percentage change rates of total cost reduction and begin a steady downward trend, slightly below $1.5 \%$.

We continue to perform reliability parameter analysis based on each model so as to identify the specific range significantly affected by decision preferences, like confidence level and load-shedding loss allowance. Figure 4 shows the percentage change rate of total cost increment as percentage loss allowance is increased (i.e., $\frac{\partial z^{*}}{\partial \theta}(\bar{\phi})$ ), where four models are displayed in the subgraphs individually. Each type of line with a specific marker represents a confidence level. Although the different confidence levels show their own volatilities, 
the significant percentage change rates based on each model generally lie between specific loss allowance. While increasing the percentage loss allowance, the SUCR has a wide volatile range covering percentage loss allowance from $8 \%$ to $20 \%$, where the confidence level makes a big difference among the lines. The SUCR-DR model has a similar volatile range. While the obvious volatile range for SUCR-ES stays below $10 \%$, and the SUCR-DR-ES volatile range is between $14 \%$ and $20 \%$. Therefore, these percentage change rate results demonstrate that different models have their own active cost increasing ranges, which are highly dependent on the chosen loss allowance and confidence level. Meanwhile, if the low-level loss allowance $(<10 \%)$ is selected, any non-generation resources can keep the total generation cost increments at a lower level and maintain the relatively steady generation costs. In particular, the percentage change rate of SUCRDR-ES for different confidence levels have less variations until the percentage loss allowance rises to $14 \%$. It again confirms that it is capable of the least-cost generations given the same level of reliability.

Figure 5 explicitly displays the relationship between the optimal objective value and the percentage change rate of total cost increment. The percentage of objective value is used to represent the current level of an objective value given the specific reliability parameter, which is divided by the highest point of objective value at $\theta=99 \%$ and $\bar{\phi}=0$. We here choose the SUCR-DR model and the SUCR-ES model to illustrate the effects of confidence level on the optimal objective value as well as the percentage change rate of total cost increment corresponding to individual non-generation resource. We observe that the $75 \%$ confidence level is likely a threshold since the objective values have explicit increases in both models and the the percentage change rates over $75 \%$ confidence level rise to relatively higher levels. If less than $75 \%$ confidence level is selected, these two resources are not able to reduce the generation costs significantly but sacrifice the system's reliability quite a lot. Therefore, it's more sensitive and reasonable to control the load-shedding loss risks by selecting a much higher confidence level.

\subsection{Enhanced 118-Bus System}

The IEEE 118-bus system has been widely used to verify the adaptability and effectiveness of proposed models (e.g., [5, 17]) and further test the performance of proposed algorithms (e.g., the Sampling Average Approximation method [23] and Benders' Decomposition [41]). To adopt high renewable penetration and non-generation resources to current power networks, we added new features on the original IEEE 118bus system, by including renewable energy resources, adjusting demand locations, setting energy storage locations and restricting transmission line capacities. An enhanced IEEE 118-bus system is used to test the proposed models for the comparisons of confidence level and loss allowance on generation cost. The system has 54 thermal units, 186 transmission lines and 103 demand sides. The total peak load from benchmark demand is $6961 \mathrm{MW}$ and occurs at hour 19. The renewable energy resources are located at Bus 1, 9, 10 and 12; meanwhile, the renewable energy output at each bus is based on the same normal distribution with 7-Bus system but different data patterns. There are 100 scenarios generated for renewable energy supply, electricity price and price elasticity, respectively. Due to the physical memory limitation, Bender's decomposition is applied to solve SUCR-DR-ES model with larger numbers of scenarios. The computational time for the SUCR-DR-ES model with 100 scenarios generally is around 60 minutes on a $2.8 \mathrm{GHz}$ PC with $8 \mathrm{~GB}$ memory, which verifies that the modified Bender's decomposition can effectively solve the 118-bus system within reasonable computation times.

We run the four models by adjusting loss allowance $\bar{\phi}$ with $1 \%$ increment and then the percentages of cost reduction are plotted in Figure 6. While selecting the reliability requirements, the SUCR-DR-ES model can achieve the largest generating cost reduction, followed by SUCR-ES, SUCR-DR and SUCR. As the loss allowance increases, the abilities of cost reduction from SUCR-ES doesn't show an absolute advantage over SUCR-DR, shown in Figure 7. If the loss allowance is less than or equal to 6\%, SUCR-ES is able to decrease total generation costs up to $1 \%$; If the loss allowance rises over 7\%, SUCR-DR appears to 
be slightly more cost-efficient than SUCR-ES, with the difference between them being no more than $0.3 \%$. However, SUCR-DR-ES still provides the minimum objective costs in all instances. This observation again indicates that the ability of SUCR-DR-ES model to reduce generating costs is apparently superior to the other models. In other words, the operation including both DR and ES is more attractive and competitive in the short-term operation.

\section{Conclusion}

A two-stage risk-constrained stochastic unit commitment model considering non-generation resources and risk control has been developed in this paper. Compared to the SUCR model, SUCR-DR model and SUCR-ES model, the numerical experiment results verified that the SUCR-DR-ES model yields the optimal UC schedule with the lowest total expected costs. When the same uncertainties occur, the models with nongeneration resources appear more stable and flexible to supply-demand changes, compared to the basic model (SUCR). With growing renewable energy penetration, the combination of demand response and energy storage provides a promising opportunity to improve the efficiency and reliability of power systems.

In the proposed models, risk management on load-shedding losses is employed by introducing CVaR constraints to the traditional two-stage stochastic UC models. On one hand, this approach helps the ISOs control the risks resulting from the load-shedding losses under different instances. On the other hand, CVaR constraints can keep the stochastic MILP models favorable for computation. In addition, this paper focuses on the reliability parameter analysis regarding confidence level and load-shedding allowance based on the percentage change rates of total cost. Conservative decisions (higher confidence level or lower loss allowance) usually means high cost increment. In addition to such findings, we found that the confidence level dominates the cost increase, but loss allowance is a relatively more significant factor leading to the magnitude of total cost increment percentage change rate. From the reliability parameter analysis, we found out the cost sensitivity of different models with the reliability parameter changes. With fixed resources, we explicitly show how the specific range of reliability parameters can affect the optimal costs. In the 118Bus system, the individual non-generation resources become less beneficial to the system cost reduction. However, considering the optimal cost and risk resilience, the model with both non-generation resources is still equipped with the strongest ability to save generation costs and maintain the power system reliability.

\section{Acknowledgement}

The works of the first and the second author are in part supported by the National Science Foundation under grants CMMI-1355939 and ECCS-1232168. The authors also would like to thank the reviewers for their suggestions on improving the quality of this paper.

\section{NOMENCLATURE}

\section{A. Sets and Indices}

A Set of transmission lines

$G$ Set of all generators

$G_{i}$ Set of electrical power generators at bus i

$N$ Set of locations (buses) 
$T$ Length of planning horizon

$g$ Indices of generators

$i, j$ Indices of buses

$t$ Time period

$\Xi$ The set of all possible scenarios

$\xi$ Indices of scenarios

B. Parameters

$S U_{g t}$ start-up cost of unit $g$ in period $t$

$S D_{g t}$ shut-down cost of unit $g$ in period $t$

$\operatorname{Prob}^{\xi}$ probability of scenario $\xi$

$L_{g}$ minimum ON time of unit $g$

$l_{g}$ minimum OFF time of unit $g$

$P_{g}^{\max }$ maximum power generation of unit $g$

$P_{g}^{\min }$ minimum power generation of unit $g$

$R U_{g}$ ramping up limit of unit $g$

$R D_{g}$ ramping down limit of unit $g$

$R S_{i t}$ spinning reserve requirement at bus $i$ in period $t$

$S_{g}^{\max }$ maximum spinning reserve of unit $g$

$R_{i t}^{\xi}$ renewable energy at bus $i$ in period $t$ of scenario $\xi$

$D_{i t}$ forecasted demand at bus $i$ in period $t$

$E_{i t}^{\xi}$ price elasticity at bus $i$ in period $t$ of scenario $\xi$

$\rho_{i}$ storage efficiency at bus $i$

$B_{i j t}$ susceptance in branch $i-j$ in period $t$;

$\theta$ confidence level

$\beta_{i t}^{\xi}$ voltage angle at bus $i$

$\bar{\phi}$ maximum load-shedding loss allowance

$\alpha, \gamma$ price velocity indicators

$\kappa_{i}$ maximum storage capacity at bus $i$ 


\section{Variables}

$u_{g t}$ commitment decision of unit $g$ at period $t$

$v_{g t}$ startup action of unit $g$ at period $t$

$w_{g t}$ shutdown action of unit $g$ at period $t$

$p_{g t}^{\xi}$ power generation of unit $g$ in period $t$ of scenario $\xi$

$s_{g t}^{\xi}$ spinning reserve of unit $g$ in period $t$ of scenario $\xi$

$f_{i j t}^{\xi}$ power transmission from bus $i$ to bus $j$ in period $t$ of scenario $\xi$

$q_{i t}^{\xi}$ electricity price at bus $i$ in period $t$ of scenario $\xi$

$r_{i t}^{\xi}$ remaining power at bus $i$ in period $t$ of scenario $\xi$

$v_{i t}^{\xi}$ power saving at bus $i$ in period $t$ of scenario $\xi$

$x_{i t}^{\xi}$ renewable energy dispatch amount at bus $i$ in period $t$ of scenario $\xi$

$y_{i t}^{\xi}$ shifted demand at bus $i$ in period $t$ of scenario $\xi$

$\eta_{t}$ value-at-risk at period $t(\mathrm{VaR})$

$\zeta_{t}^{\xi}$ the loss exceeding VaR in period $t$ of scenario $\xi$

\section{References}

[1] R. Barth, H. Brand, P. Meibom, C. Weber, A stochastic unit-commitment model for the evaluation of the impacts of integration of large amounts of intermittent wind power, in: International Conference on Probabilistic Methods Applied to Power Systems, 2006, pp. 1-8.

[2] P. Ruiz, C. Philbrick, E. Zak, K. Cheung, P. Sauer, Uncertainty management in the unit commitment problem, IEEE Transactions on Power Systems 24 (2) (2009) 642-651.

[3] A. Tuohy, P. Meibom, E. Denny, M. O’Malley, Unit commitment for systems with significant wind penetration, IEEE Transactions on Power Systems 24 (2) (2009) 592-601.

[4] J. Wang, A. Botterud, G. Conzelmann, G. S. Vladimiro Miranda, Claudio Monteiro, Impact impact of wind power of wind power forecasting on unit commitment and dispatch, in: 8th Int. Wind Integration Workshop, Bremen, Germany, 2009.

[5] J. Wang, M. Shahidehpour, Z. Li, Security-constrained unit commitment with volatile wind power generation, IEEE Transactions on Power Systems 23 (3) (2008) 1319-1327.

[6] Q. P. Zheng, S. Rebennack, P. M. Pardalos, M. V. F. Pereira, N. A. Iliadis, Handbook of $\mathrm{CO}_{2}$ in Power Systems, Energy Systems, Springer, 2012.

[7] X. Liu, Optimization of a combined heat and power system with wind turbines, International Journal of Electrical Power \& Energy Systems 43 (1) (2012) 1421-1426.

[8] H. Daneshi, A. Srivastava, Security-constrained unit commitment with wind generation and compressed air energy storage, IET Generation, Transmission and Distribution 6 (2) (2012) 167-175.

[9] S. Chakraborty, T.Senjyu, H. Toyama, A. Saber, T. Funabashi, Determination methodology for optimising the energy storage size for power system, IET Generation, Transmission and Distribution 3 (11) (2009) 987-999.

[10] M. Xu, X. Zhuan, Identifying the optimum wind capacity for a power system with interconnection lines, International Journal of Electrical Power \& Energy Systems 51 (2013) 82-88. 
[11] S. Mohammadi, S. Soleymani, B. Mozafari, Scenario-based stochastic operation management of microgrid including wind, photovoltaic, micro-turbine, fuel cell and energy storage devices, International Journal of Electrical Power \& Energy Systems 54 (2014) 525-535.

[12] A. Rong, R. Lahdelma, P. B. Luh, Lagrangian relaxation based algorithm for trigeneration planning with storages, European Journal of Operational Research 188 (1) (2008) 240-257.

[13] C. D. Jonghe, E. Delarue, R. Belmans, W. D'haeseleer, Determining optimal electricity technology mix with high level of wind power penetration, Applied Energy 88 (6) (2011) 2231 - 2238.

[14] T. Senjyu, T. Miyagi, S. A. Yousuf, N. Urasaki, T. Funabashi, A technique for unit commitment with energy storage system, Electrical Power and Energy Systems 29 (1) (2007) 91-98.

[15] L. Sigrist, E. Lobato, L. Rouco, Energy storage systems providing primary reserve and peak shaving in small isolated power systems: An economic assessment, International Journal of Electrical Power \& Energy Systems 53 (2013) 675-683.

[16] R. Sioshansi, Evaluating the impacts of real-time pricing on the cost and value of wind generation, IEEE Transactions on Power Systems 25 (2) (2010) 741-748.

[17] L. Zhao, B. Zeng, Robust unit commitment problem with demand response and wind energy, in: IEEE Power and Energy Society General Meeting, 2012, pp. 1-8.

[18] K. Dietrich, J. Latorre, L. Olmos, A. Ramos, Demand response in an isolated system with high wind integration, IEEE Transactions on Power Systems 27 (1) (2012) 20-29.

[19] A. Kowli, S. Meyn, Supporting wind generation deployment with demand response, in: IEEE Power and Energy Society General Meeting, 2011, pp. 1-8.

[20] F. Partovi, M. Nikzad, B. Mozafari, A. M. Ranjbar, A stochastic security approach to energy and spinning reserve scheduling considering demand response program, Energy 36 (5) (2011) 3130 - 3137.

[21] S. Madaeni, R. Sioshansi, The impacts of stochastic programming and demand response on wind integration, Energy Systems 4 (2) (2013) 109-124.

[22] J. Wang, S. Kennedy, J. Kirtley, A new wholesale bidding mechanism for enhanced demand response in smart grids, in: Innovative Smart Grid Technologies, 2010, pp. 1-8.

[23] Q. Wang, Y. Guan, J. Wang, A chance-constrained two-stage stochastic program for unit commitment with uncertain wind power output, IEEE Transactions on Power Systems 27 (1) (2012) 206-215.

[24] U. A. Ozturk, M. Mazumdar, B. A. Norman, A solution to the stochastic unit commitment problem using chance constrained programming, IEEE Transactions on Power Systems 19 (3) (2004) 1589-1598.

[25] H. Yu, C. Y. Chung, K. P. Wong, J. H. Zhang, A chance constrained transmission network expansion planning method with consideration of load and wind farm uncertainties, IEEE Transactions on Power Systems 24 (3) (2009) 1568-1576.

[26] R. T. Rockafellar, S. Uryasev, Optimization of conditional value-at-risk, Journal of Risk 2 (2000) 21-42.

[27] S. Uryasev, Conditional value-at-risk: Optimization algorithms and applications, in: Computational Intelligence for Financial Engineering, IEEE, 2000, pp. 49-57.

[28] S. Sarykalin, G. Serraino, S. Uryasev, Value-at-risk vs. conditional value-at-risk in risk management and optimization, Tutorials in Operations Research.INFORMS, Hanover, MD (2008) 270-294.

[29] P. A. Ruiz, C. R. Philbrick, P. W. Sauer, Wind power day-ahead uncertainty management through stochastic unit commitment policies, in: Power Systems Conference and Exposition, 2009, pp. 1-9.

[30] E. M. Constantinescu, V. M. Zavala, M. Rocklin, S. Lee, M. Anitescu, A computational framework for uncertainty quantification and stochastic optimization in unit commitment with wind power generation, IEEE Transactions on Power Systems 26 (1) (2011) 431-441.

[31] A. Papavasiliou, S. S. Oren, R. P. O'Neill, Reserve requirements for wind power integration: A scenario-based stochastic programming framework, IEEE Transactions on Power Systems 26 (4) (2011) 2197-2206.

[32] Q. P. Zheng, J. Wang, P. M. Pardalos, Y. Guan, A decomposition approach to the two-stage stochastic unit commitment problem, Annals of Operations Research 210 (2013) 387-410.

[33] D. S. Kirschen, G. Strbac, P. Cumperayot, D. de Paiva Mendes, Factoring the elasticity of demand in electricity prices, IEEE Transactions on Power Systems 15 (2) (2000) 612-617.

[34] J. Wang, S. W. Kennedy, J. L. Kirtley, Optimization of forward electricity markets considering wind generation and demand response, IEEE Transactions on Smart Grid 5 (3) (2014) 1254-1261.

[35] Q. Wang, J. Wang, Y. Guan, Stochastic unit commitment with uncertain demand response, IEEE Transactions on Power Systems 28 (1) (2013) 562-563.

[36] G. J. Alexander, A. M. Baptista, A comparison of VAR and CVaR constraints on portfolio selection with the mean-variance model, Management Science 50 (9) (2004) 1261-1273.

[37] Q. P. Zheng, P. M. Pardalos, Stochastic and risk management models and solution algorithm for natural gas transmission network expansion and lng terminal location planning, Journal of Optimization Theory and Applications 147 (2010) 337 357. 
[38] Q. P. Zheng, S. Shen, Y. Shi, Loss-Constrained Minimum Cost Flow under Arc Failure Uncertainty with Applications in Risk-Aware Kidney Exchange, Submitted.

[39] M. Shahidehopour, Y. Fu, Benders decomposition: applying benders decomposition to power systems, IEEE Power and Energy Magazine 3 (2) (2005) 20-21.

[40] H. Siahkali, M. Vakilian, Stochastic unit commitment of wind farms integrated in power system, Electric Power Systems Research 80 (9) (2010) 1006-1017.

[41] C. Zhao, J. Wang, J. P. Watson, Y. Guan, Multi-stage robust unit commitment considering wind and demand response uncertainties, IEEE Transactions on Power Systems 28 (3) (2013) 2708-2717. 


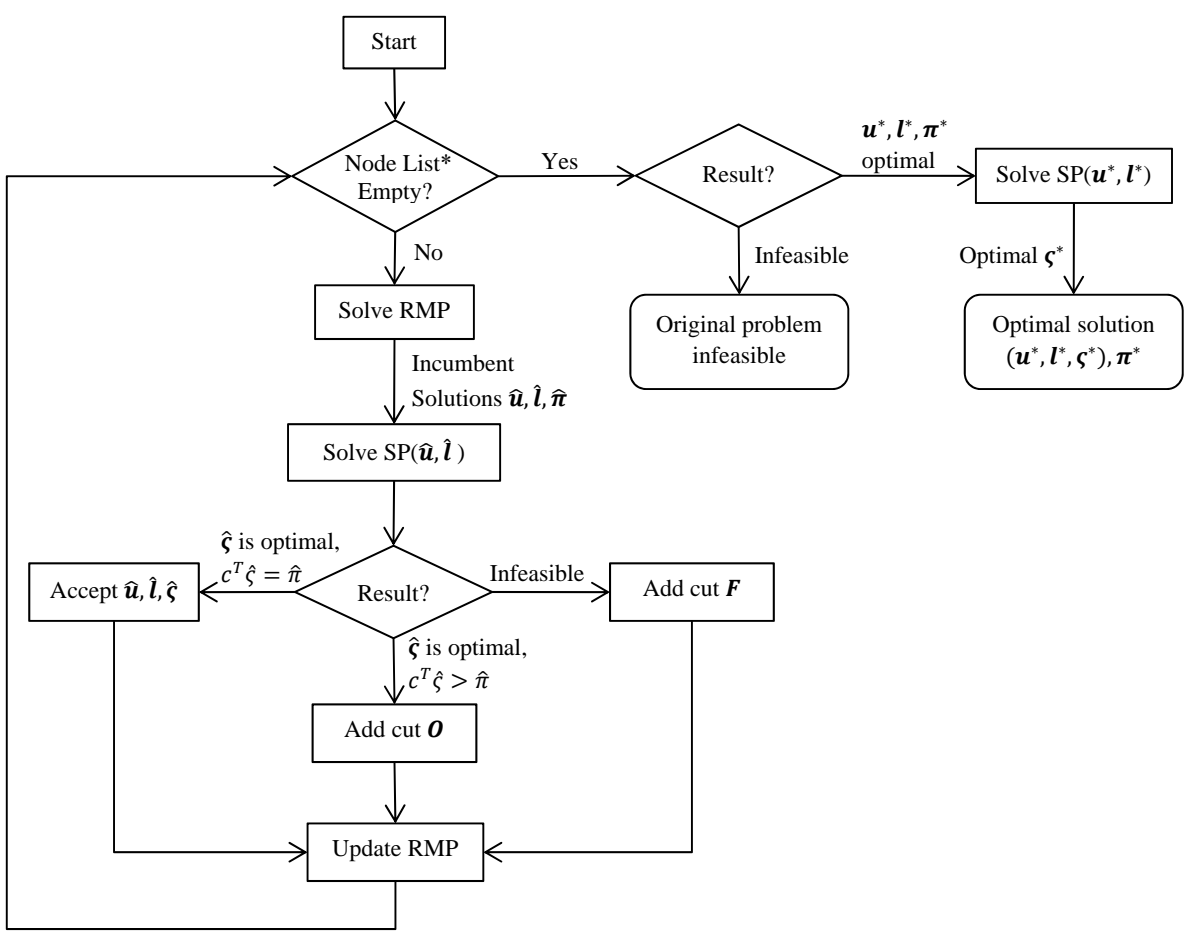

* Brand-and-Bound node list

Figure 1: The solution flowchart of Benders' Decomposition with CALLBACK function

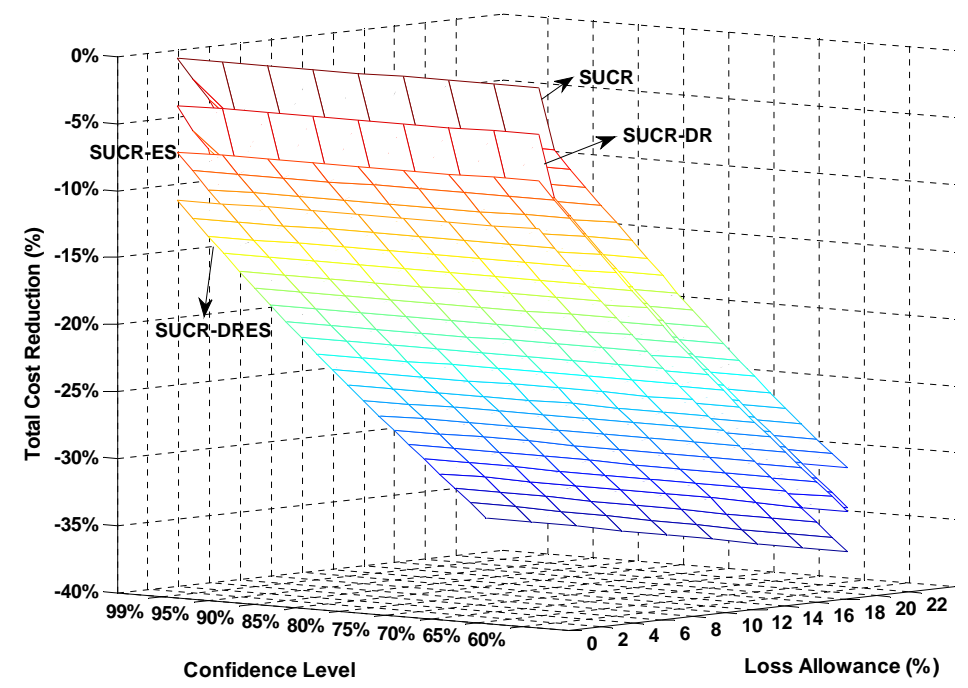

Figure 2: Cost Saving Comparisons in Three-Dimension (7-Bus System) 
Table 1: Bus Parameters

\begin{tabular}{c|c|c|c|c}
\hline ID & Type & Gen ID & $\begin{array}{c}\text { Gen. Cap. } \\
\text { (MW) }\end{array}$ & $\begin{array}{c}\text { Strg. Cap. } \\
\text { (MW) }\end{array}$ \\
\hline B1 & Renewable & R1 & 100 & 80 \\
B2 & Coal & G1 & 110 & 20 \\
B3 & Coal & G2 & 50 & - \\
B4 & Gas & G3 & 90 & 20 \\
B5 & - & - & - & 10 \\
B6 & - & - & - & - \\
B7 & Coal & G4 & 70 & - \\
\hline
\end{tabular}

a The symbol,'-', represents no generation unit available at a corresponding bus

Table 2: Generator Parameters

\begin{tabular}{l|c|c|c|c}
\hline & G1 & G2 & G3 & G4 \\
\hline Min-ON (h) & 2 & 1 & 2 & 4 \\
Min-OFF (h) & 2 & 2 & 2 & 1 \\
Ramp-Up (MW/h) & 60 & 30 & 60 & 60 \\
Ramp-Down (MW/h) & 60 & 30 & 60 & 60 \\
Pmin (MW) & 10 & 5 & 9 & 7 \\
Pmax (MW) & 110 & 50 & 90 & 70 \\
Max-Spn (MW) & 20 & 20 & 15 & 15 \\
Required Spn (MW) & 10 & 0 & 0 & 0 \\
\hline
\end{tabular}

Table 3: Generation Cost Parameters

\begin{tabular}{l|c|c|c|c}
\hline & G1 & G2 & G3 & G4 \\
\hline Startup $(\$)$ & 50 & 500 & 800 & 30 \\
Shutdown $(\$)$ & 50 & 500 & 800 & 20 \\
Fuel Cost $a(\$)$ & 6.78 & 6.78 & 31.67 & 10.15 \\
Fuel Cost $b(\$ / M W h)$ & 12.888 & 12.888 & 26.244 & 17.820 \\
Fuel Cost $c\left(\$ / M W h^{2}\right)$ & 0.0109 & 0.0109 & 0.0697 & 0.0128 \\
\hline
\end{tabular}


Table 4: Transmission Line Parameters

\begin{tabular}{c|c|c|c|c}
\hline ID & From & To & Flow Capacity(MW) & Voltage(V) \\
\hline L1 & B1 & B2 & 50 & 500 \\
L2 & B1 & B3 & 160 & 500 \\
L3 & B1 & B4 & 80 & 500 \\
L4 & B2 & B3 & 100 & 500 \\
L5 & B2 & B5 & 50 & 500 \\
L6 & B3 & B5 & 30 & 500 \\
L7 & B3 & B6 & 100 & 500 \\
L8 & B4 & B6 & 50 & 500 \\
L9 & B4 & B7 & 60 & 500 \\
L10 & B6 & B7 & 50 & 500 \\
\hline
\end{tabular}

Table 5: Optimal Unit Commitment For 7-Bus System

\begin{tabular}{|c|c|c|c|}
\hline Model Type & Objective Value & Unit ID & Hour (1-24) \\
\hline \multirow{4}{*}{ SUCR } & \multirow{4}{*}{$\$ 54917.9$} & G1 & 1111111111111111111111 \\
\hline & & G2 & 111111111111111111111111 \\
\hline & & G3 & 000000000000000000000000 \\
\hline & & G4 & 1111111111111111111111111111 \\
\hline \multirow{4}{*}{ SUCR-DR } & \multirow{4}{*}{$\$ 52758.6$} & G1 & 111111111111111111111111 \\
\hline & & $\mathrm{G} 2$ & 111111111111111111111111 \\
\hline & & G3 & 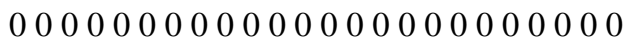 \\
\hline & & G4 & 100111111111111111111111 \\
\hline \multirow{4}{*}{ SUCR-ES } & \multirow{4}{*}{$\$ 52594.6$} & G1 & 111111111111111111111111 \\
\hline & & $\mathrm{G} 2$ & 111111111111111111111111 \\
\hline & & G3 & 000000000000000000000000 \\
\hline & & G4 & 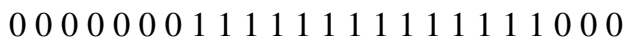 \\
\hline \multirow{4}{*}{ SUCR-DR-ES } & \multirow{4}{*}{$\$ 50480.5$} & G1 & 111111111111111111111111 \\
\hline & & $\mathrm{G} 2$ & 111111111111111111111111 \\
\hline & & G3 & 000000000000000000000000 \\
\hline & & G4 & 000000011111111111110000 \\
\hline
\end{tabular}




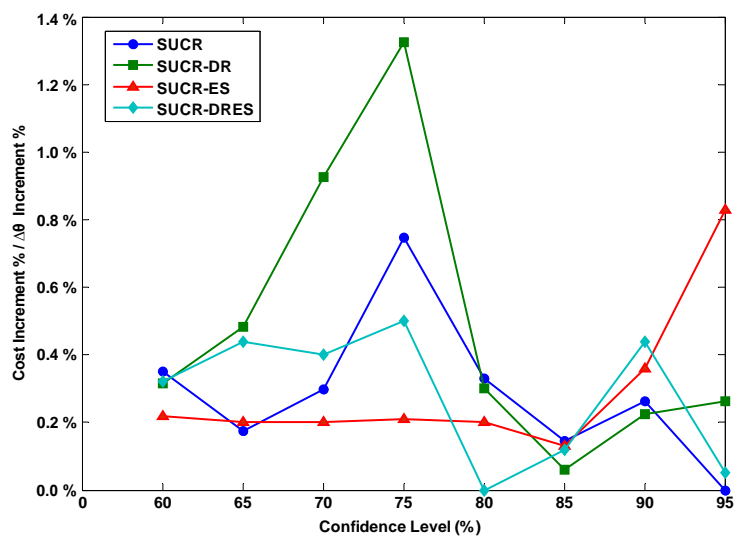

(a) Confidence Level at $\bar{\phi}=10 \%$

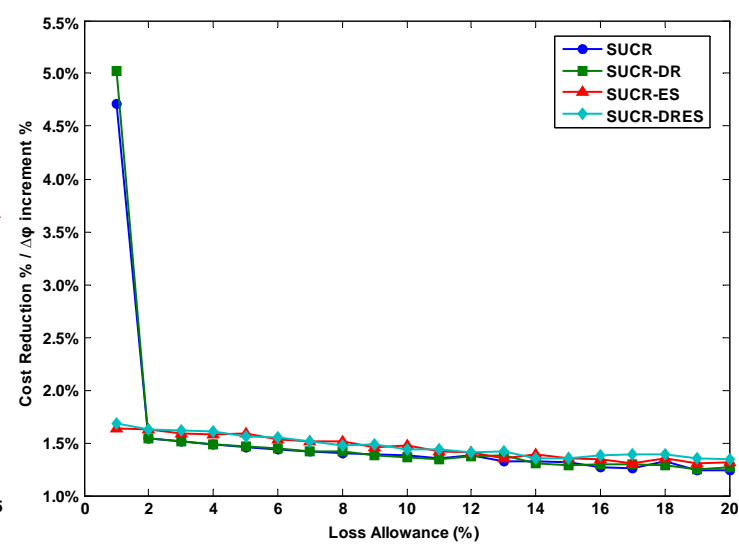

(b) Loss Allowance at $\theta=90 \%$

Figure 3: Comparisons of the Percentage Change Rates at Confidence Level and Load-Shedding Loss Allowance, Respectively 


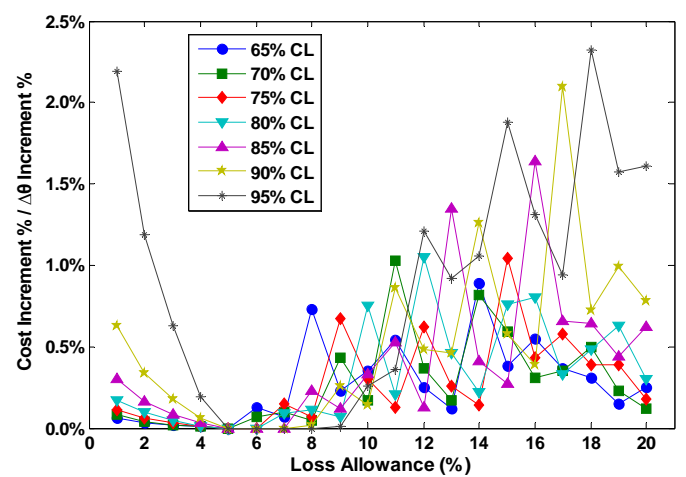

(a) SUCR Model

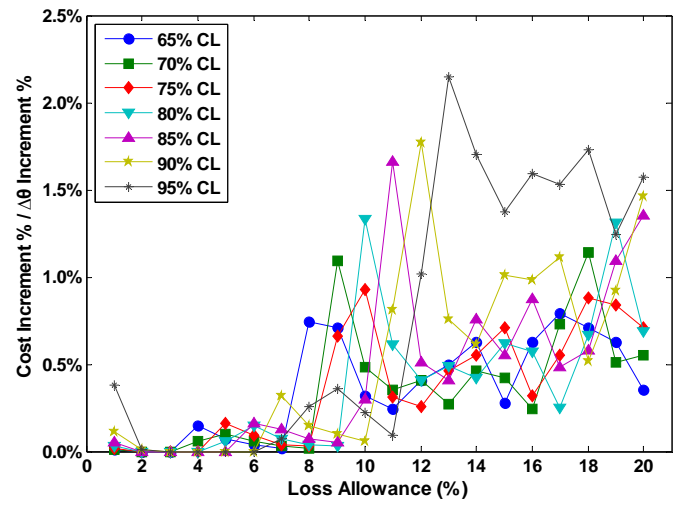

(b) SUCR-DR Model

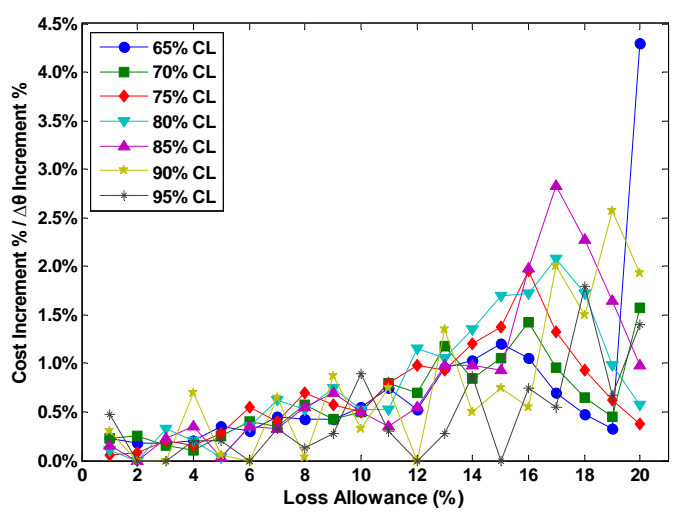

(c) SUCR-ES Model

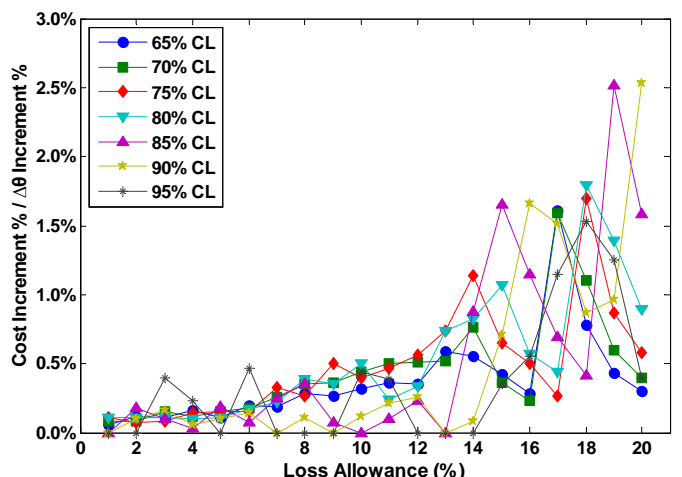

(d) SUCR-DR-ES Model

Figure 4: Reliability Parameter Analysis for Each Model 

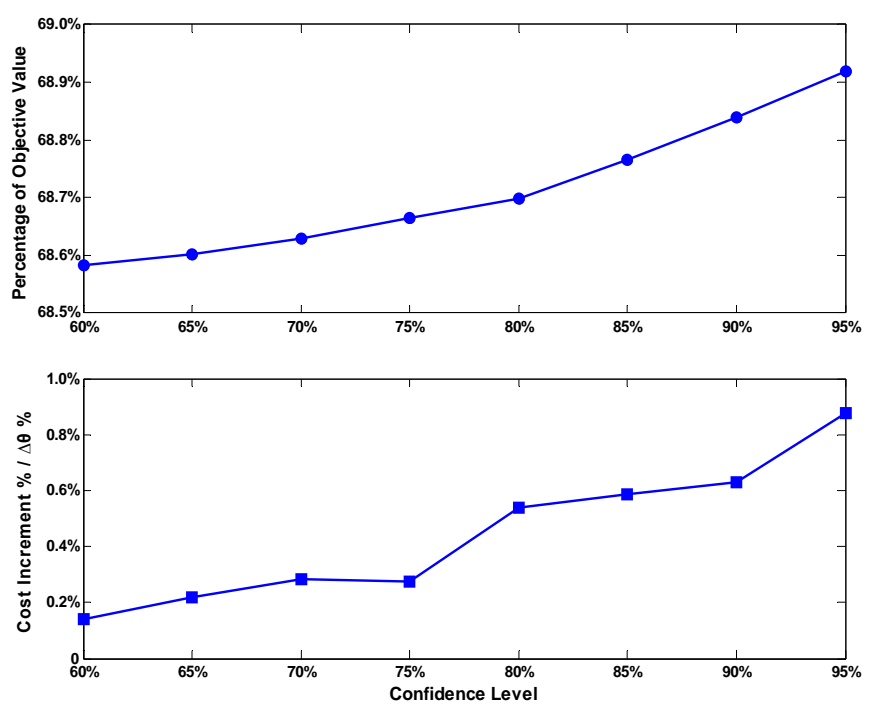

(a) SUCR-DR Model
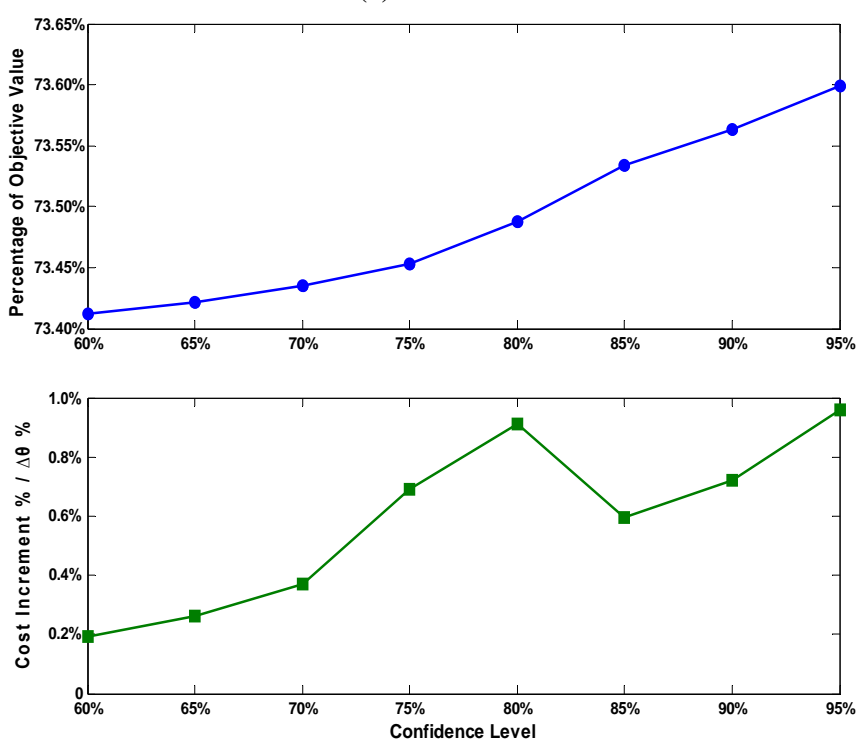

(b) SUCR-ES Model

Figure 5: Comparisons of Objective Values and Percentage Change Rates at Confidence Level 


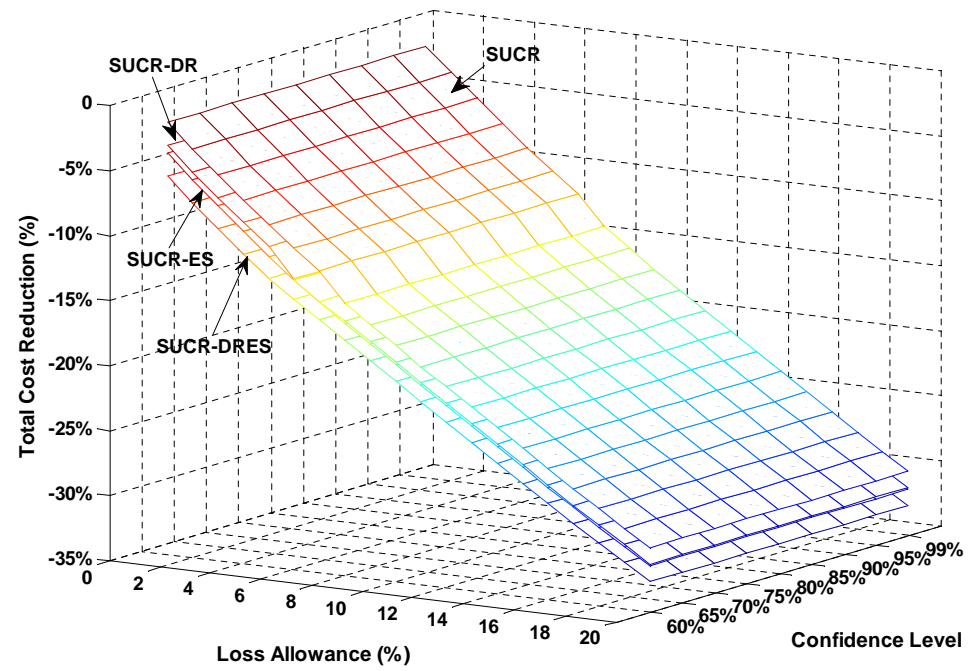

Figure 6: Cost Saving Comparisons in Three-Dimension (118-Bus System)

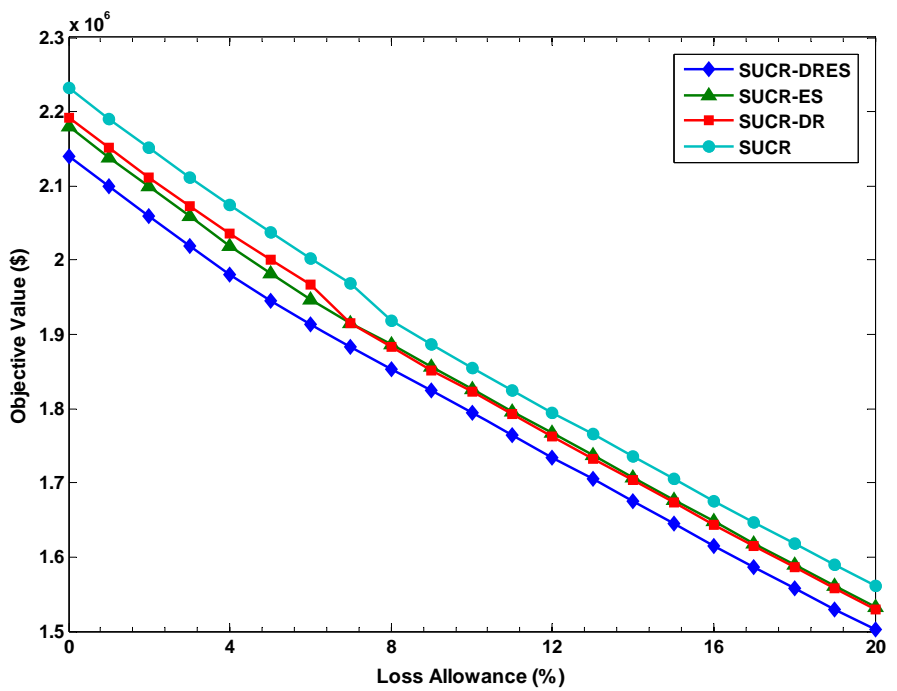

Figure 7: The Objective Value v.s. Loss Allowance (118-Bus System) 